\title{
EDITORIAL
}

\section{Pericyclic reactions in the chemistry of heterocycles}

Pericyclic reactions are characterized with cyclic transition states, resulting in high regio- and stereoselectivity, thus are widely used as synthetic tools for the construction of complex molecular frameworks of natural products and for the preparation of heterocyclic compounds containing aromatic, as well as non-aromatic rings. Such reactions, as a rule, have low energy barriers and proceed under relatively mild conditions. The main characteristics of pericyclic processes in purely hydrocarbon systems can be reliably predicted by the Woodward-Hoffmann rules.

The introduction of heteroatoms in the conjugated system of pericyclic reaction substrates significantly expands the range of synthetic applicability of these reactions. Cycloaddition and electrocyclization reactions are among the most significant tools for the synthesis of heterocycles, which have been used for the preparation of various heterocyclic compounds usually containing between 3 and 7 atoms in the ring. The introduction of heteroatoms in the conjugated system leads to polarization of molecules and alters the energy of orbitals. This, in turn, may dismiss the forbidden nature of certain reactions. When heteroatoms are present in the terminal positions, the cyclization mechanism may change and the reaction may occur by pseudopericyclic (heteroelectrocyclic) mechanism. For this reason, it is important to study the reactions in conjugated systems containing heteroatoms, both for the advancement of heterocyclic synthesis and for the identification of new pericyclic reaction mechanisms.

This thematic issue of the journal has been dedicated to pericyclic reactions in systems containing heteroatoms, applicable to the synthesis and transformations of heterocycles, and includes 8 reviews, 9 experimental and 2 theoretical articles.

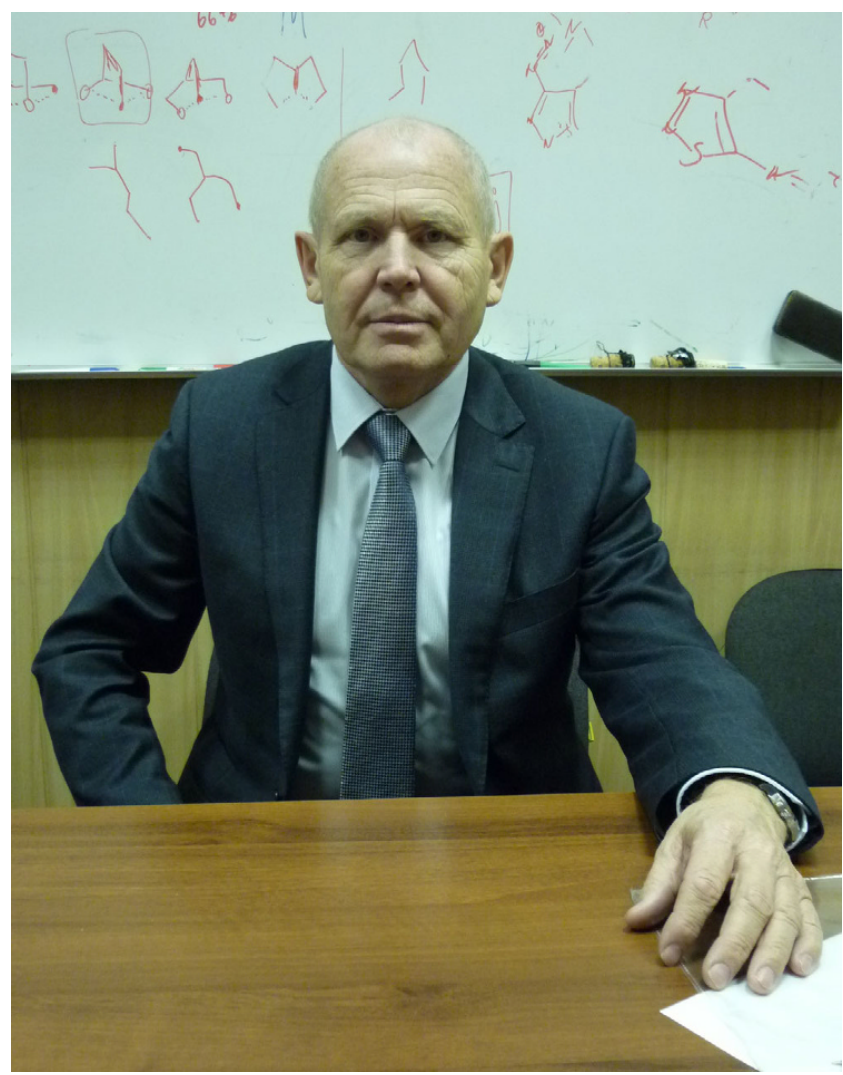

I am convinced that chemists working in the field of heterocyclic chemistry will find in this issue new and useful information and will gain new impetus for achieving progress in their fields of study.

On behalf of editorial board and myself, I would like to express my gratitude to the authors for their contributions. Particular recognition is deserved by the esteemed reviewers and editorial staff, who carefully "polished" the manuscripts and ensured that publication deadlines were met. We sincerely hope that the authors will in the future again choose the journal "Chemistry of Heterocyclic Compounds" for the publication of their further discoveries.

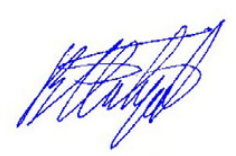

Editor of the thematic issue, Professor, Dr. Chem. V. A. Bakulev, Ural Federal University named after the first President of Russia B. N. Yeltsin 